\title{
MP
}

GMR

\section{Establishment of a neuroblastoma mouse model by subcutaneous xenograft transplantation and its use to study metastatic neuroblastoma}

Q. Gao ${ }^{1 *}$, C.F. Chen ${ }^{2 *}$, Q. Dong ${ }^{3}$, L. Hou ${ }^{4}$, X. Chen ${ }^{3}$, Y.L. Zhi ${ }^{3}$, X. Li ${ }^{3}$, H.T. Lu ${ }^{3}$ and H.Y. Zhang ${ }^{3}$

'Department of Pediatric Surgery, Qingdao Women and Children Hospital, Qingdao, Shandong, China

2Department of Pediatric Surgery, Linyi People's Hospital, Shandong, China

${ }^{3}$ Department of Pediatric Surgery, The Affiliated Hospital of Qingdao University,

Qingdao, Shandong, China

${ }^{4}$ Department of Biochemistry, Medical College, Qingdao University, Qingdao,

Shandong, China

*These authors contributed equally to this study.

Corresponding author: H.T. Lu

E-mail: leihanleihan@126.com

Genet. Mol. Res. 14 (4): 16297-16307 (2015)

Received June 12, 2015

Accepted September 2, 2015

Published December 8, 2015

DOI http://dx.doi.org/10.4238/2015.December.8.20

ABSTRACT. The aim of this study was to establish a metastatic human neuroblastoma (NB) mouse model by xenograft in order to study the metastatic mechanisms of NB. A human NB cell line was obtained from a 5-year-old patient and cultured in vitro. A suspension of these cells was subcutaneously inoculated into nude mice at the right flank next to the forelimb. The biological characteristics of the developed subcutaneous and metastatic tumors were analyzed by hematoxylin and eosin staining. The expression of the tumor marker neuron-specific enolase was determined by immunohistochemistry, and the invasive ability of metastatic tumors 
was examined by a Matrigel invasion assay. DNA microarray analyses were performed to examine the metastasis-related gene expression. Our results showed that tumors grew in $75 \%$ of the mice injected with NB cells and the rate of metastasis was $21 \%$. The xenograft tumors retained the morphological and biological characteristics of the NB specimen from the pediatric patient. Neuron-specific enolase was highly expressed in both subcutaneous and metastatic tumors. The metastatic tumor cells possessed a higher invasive capability than the primary NB cells. The expression of 25 metastasis-related genes was found to be significantly altered in metastatic tumors compared to primary tumors, including RECK, MMP2, VEGF, MMP3, and CXCL12. In conclusion, we successfully established a human NB xenograft model with high tumor-bearing and metastatic rates in nude mice, providing an ideal animal model for the in vivo study of NB.

Key words: Neuroblastoma; Metastasis; Nude mice; Xenograft; Neuron-specific enolase

\section{INTRODUCTION}

Metastasis is one of the biological characteristics of malignant tumor cells and is a major cause of cancer mortality (Yan et al., 2013). A complete understanding of the mechanisms underlying metastasis is critical for prognosis and the development of new treatment options. Neuroblastoma (NB) is one of the most common malignant pediatric tumors (Ara and DeClerck, 2006; Navarro et al., 2012). It is characterized by early metastasis in the lung, bone, brain, and bone marrow (Redlinger et al., 2004; Ara and DeClerck, 2006; Navarro et al., 2012), and this characteristic distinguishes NB from other malignant tumors and is a major cause of death in patients (Redlinger et al., 2004). Therefore, further study of the underlying mechanism, influential factors, and potential preventive and therapeutic methods of metastasis in NB has become a primary focus of NB research. Neuron-specific enolase (NSE) is a glycolytic enzyme expressed in the neuroendocrine system (Cooper, 1994). Elevated expression of NSE is closely associated with malignant tumors such as NB and small-cell lung carcinoma (SCLC). NSE is a tumor marker of pediatric NB and is often used in prognosis and follow-up (Cooper, 1994; Abdennebi et al., 2000).

Establishing animal models of metastatic human tumors by xenograft is an important tool in the study of tumor metastasis and the identification of experimental therapeutics for its treatment (Nofiele and Cheng, 2014). Nude mice are ideal models to study tumor metastasis, as the methods are simple with high success rates and the animals do not need pretreatment. The xenograft tumors do not attach to the skin or subcutaneous tissues, which makes it easy to isolate them for observation, measurement, and biopsy tests. The xenograft tumors also retain the morphological and biological characteristics of their corresponding original tumors. Chromosome analysis of tumor cells has shown that the tumors also retain the same karyotype as the original tumor after passage. Other isoenzymatic and immunological analyses have demonstrated that human malignant tumors retain the characteristics of the malignant tumors after xenograft and passages (Wei et al., 1996). Successful transplantation of human tumors into nude mice makes it possible to systemically study the biological characteristics of human tumors, particularly in metastatic cancers. However, earlier studies have shown that the majority of human malignant tumors have limited growth in mice and 
are rarely metastatic. These studies have focused on the identification of spontaneously occurring highly metastatic cancers. It is essential to overcome the obstacle of limited metastasis in mice in order to systemically study human tumor metastasis in non-human in vivo models (Wu et al., 1989).

By using a previously established Chinese human NB cell line, we prepared and subcutaneously injected a suspension of the tumor cells into the right forelimb of nude mice (Hao et al., 2005). The biological characteristics of the subcutaneous and metastatic tumors were observed, and histopathological and microarray analyses were performed. The expression of NSE was analyzed in the subcutaneous tumors from the nude mice and the primary NB tumor tissues from a pediatric patient. Our experiments established an animal model for the study of NB etiology, the mechanism underlying its metastasis, and approaches for experimental therapeutic treatment.

\section{MATERIAL AND METHODS}

\section{Clinical data}

The primary NB cells were obtained from a 5-year-old male patient. The pediatric patient was admitted to the hospital due to pain in the right leg for 5 days and pain in the chest for 3 days. Upon examination, he was found to have sternum tenderness. The plain film chest $x$-ray showed "mediastinal space occupying lesions", and the bone scan showed multiple systemic bone metastases, suggesting neurogenic tumors. A tumor resection was performed, and the pathological examination confirmed a diagnosis of Evans stage IV NB with systemic bone marrow metastases. A fresh tumor specimen was retrieved in surgery and cultured in vitro, and a cell line was established that has been passaged more than 216 times (Liu et al., 2011).

\section{Experimental animals}

A total of 48 SPF female BalbPc-nu mice (4 weeks old and with body weights of 12-14 g) were purchased from the Shanghai Slac Laboratory Animal Company (previously known as Shanghai Laboratory Animal Center, Chinese Academy of Sciences, China). These mice were housed in a barrier facility (specific-pathogen-free room). Food, water, bedding materials, and cages were all autoclaved before use. The specific-pathogen-free laboratory animal operation procedures were strictly followed.

\section{Cell culture}

The Chinese NB cell line was established in our lab (Hao et al., 2005). Cells were cultured in DMEM (Gibco, USA) supplemented with 10\% (v/v) fetal bovine serum (Hyclone, USA). Cells undergoing exponential growth were digested with $0.25 \%$ trypsin with $0.01 \%$ EDTA and then harvested and resuspended in serum-free culture medium. The cell density was adjusted to $1 \times 10^{8} / \mathrm{mL}$.

\section{Establishment of the metastatic tumor-bearing NB model in nude mice}

Cells undergoing exponential growth with good morphology were harvested and inoculated into nude mice. Briefly, NB cells were digested and harvested at $300 \mathrm{xg}$ for 5-8 min and 
the supernatant was discarded. The recovered cells were counted and resuspended in DMEM culture medium supplemented with 10\% newborn bovine serum (PAA, Austria) at a density suitable for inoculation. In a cell culture hood, each mouse was subcutaneously injected in the right flank near the forelimb with $1 \times 10^{7}$ cells suspended in a total volume of $0.1 \mathrm{~mL}$.

\section{Observation of the established animal model}

The injection sites were checked every day after inoculation for the appearance of ulcers, redness, and swelling. Subcutaneous nodules with a diameter exceeding $0.5 \mathrm{~cm}$ were considered to be tumors. Once the tumor-bearing nude mice were sacrificed, tumor morphology and metastasis in the intestine, liver, and peritonea were examined. The fresh tumor tissues were used for histological and immunohistochemical examinations. Subcutaneous tumors and internal organs were fixed with $10 \%$ formaldehyde solution and then embedded in paraffin. Tissue sections were stained with hematoxylin and eosin ( $\mathrm{HE})$, and the pathological morphology of the subcutaneous tumors and metastatic foci was examined under a light microscope.

\section{Immunohistochemical analysis}

NSE expression in the tumor-bearing tissues from nude mice and in the primary NB tissues from the pediatric patient were analyzed by immunohistochemical staining with anti-NSE antibody (ab53025, Abcam, UK) at 1:100 dilution. Positive NSE protein expression appeared as a dense brown color and was mainly located on the cell membrane; occasionally, positive protein expression was found in the cytoplasm.

\section{Matrigel invasion assay}

The upper chamber of a transwell ( $8 \mu \mathrm{m}$ pore size, Millipore, MA, USA) was coated with 1:6 diluted Matrigel (about $75 \mu \mathrm{g}$ for each well) and placed at $37^{\circ} \mathrm{C}$ for $5 \mathrm{~h}$ to facilitate Matrigel solidification. Then, $400 \mu \mathrm{L}$ of the cells at a concentration of $6 \times 10^{4} / \mathrm{mL}$ was added into the upper chamber, and the lower chamber was filled with $600 \mu \mathrm{L}$ DMEM culture medium containing with $10 \%$ bovine serum. After an incubation period of $48 \mathrm{~h}$ at $37^{\circ} \mathrm{C}$ in an incubator with $5 \% \mathrm{CO}_{2}$, the cells on the upper surface of the membrane were removed with a cotton swab and the cells on the bottom surface of the membrane were fixed with $4 \%$ paraformaldehyde for 30 min before staining with $0.1 \%$ crystal violet solution. Stained cells were counted in five randomly selected microscopic fields. Experiments were performed three times independently in triplicate.

\section{Detection of gene expression}

Gene expression in primary and metastatic tumors in the tumor-bearing mouse models were examined using Oligo Tumor Metastasis Microarray (SuperArray, Bethesda, MD, USA). A total of 4 gene chips were used: one for primary tumor samples and three for metastatic tumor samples. By detecting the fluorescence signals and then comparing and analyzing the images and data, the common genes with differential expression in primary and metastatic tumors were determined. The TrueLabeling-AMP linear RNA amplification kit and the OHS-028 human tumor metastasis microarray were purchased from SuperArray. 


\section{Ethics statement}

We confirmed all the protocols were performed in accordance with the approval from the authors' institutional Ethics Committees.

\section{Statistical analysis}

Statistical analysis was performed with the SPSS 12.0.0 software (Chicago, IL, USA). The comparisons between two groups were conducted using the Student $t$-test with $\mathrm{P}<0.05$ considered to be significant.

\section{RESULTS}

\section{Establishment of metastatic NB mouse model}

NB cells were inoculated into nude mice. The tumor latency was $30-35$ days with a $75 \%$ success rate for tumor production (Table 1; Figure 1A, B and C). Tumors appeared as spherical or nodular masses of soft tissue with high tension. Tumor volume gradually increased over time, and tumors became denser with a connective tissue capsule and a clear boundary. The cutting surface exhibited a light white color. During tumor formation, no spontaneous regression of the tumor was observed. The tumor-bearing nude mice appeared significantly thinner with reduced activities and responsiveness at later stages, and they developed cachexia until death from systemic failure. Among the tumor-bearing animals, there were 5 cases with local tumors of a large size that composed up to a half of the body weight; however, no metastasis occurred in these mice. In 4 cases, no tumor growth was observed at the injection; however, systemic metastasis was found in these mice. Six mice were found to develop both local and metastatic tumors, giving a metastatic rate of $21 \%$ (results summarized in Table 1 ).

Table 1. Subcutaneous and metastatic tumor formation in nude mice inoculated with NB cell suspension.

\begin{tabular}{lccc}
\hline & Subcutaneous tumor present & Subcutaneous tumor absent & Total \\
\hline With distant metastasis & 6 & 4 & $10^{\mathrm{a}}$ \\
No distant metastasis & 30 & 8 & 38 \\
Total & $36^{\mathrm{b}}$ & 12 & 48 \\
\hline
\end{tabular}

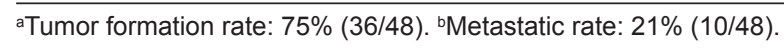
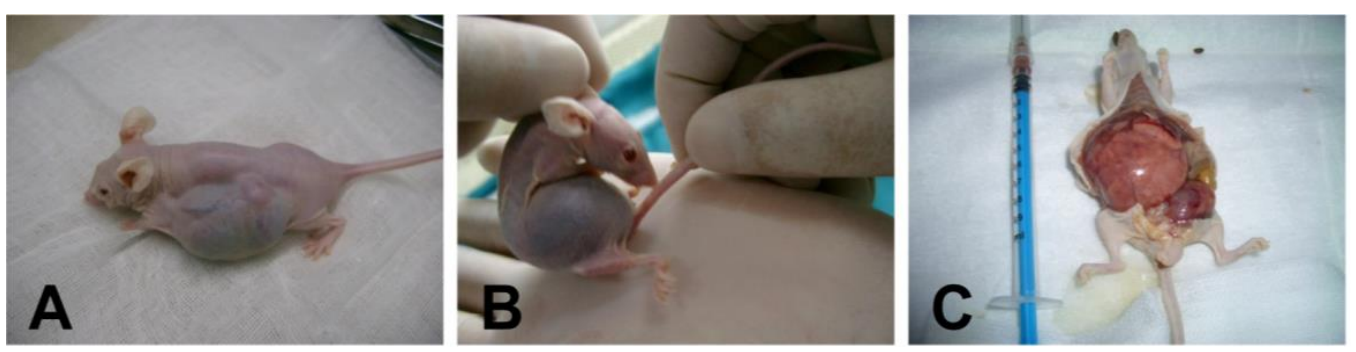

Figure 1. Establishment of a neuroblastoma (NB) mouse model. A. Tumor formation; B. peritoneal metastasis; C. extensive metastasis of NB in the liver, lung and peritoneum in a nude mouse inoculated with NB cells from a pediatric NB patient. 


\section{Histopathological examination of primary and metastatic NB tumors}

Under a light microscope, the tumor cells from both the primary NB cells obtained from the patient and the subcutaneous tumors in nude mice were diffusely distributed with large darkly stained nuclei (Figure 2A). The nuclei were round, ovular or irregularly shaped, had uneven nuclear chromatin, and occasionally had nucleoli; the cytoplasmic volume was also low in these tumor cells. Xenograft tumors were solid tumors and mostly ovular in shape. As the tumors grew, they gradually became irregularly shaped with nodular or lobulated protrusions on the surface, which was pink in color, and some had an intact capsule. The metastatic tumors were variable in size and had a scattered distribution (Figure 2B, C, and D). Under the microscope, the tumor cells were polygonal or round, large in size and had clear cytoplasm. These tumor cells showed large densely stained nuclei located at the center of the cells. The cells were disorganized and arranged as dense nests and cords, which is one of the characteristics of NB histopathology. The cells in metastatic tumors and subcutaneous xenograft tumors had similar morphology (Figure 2).
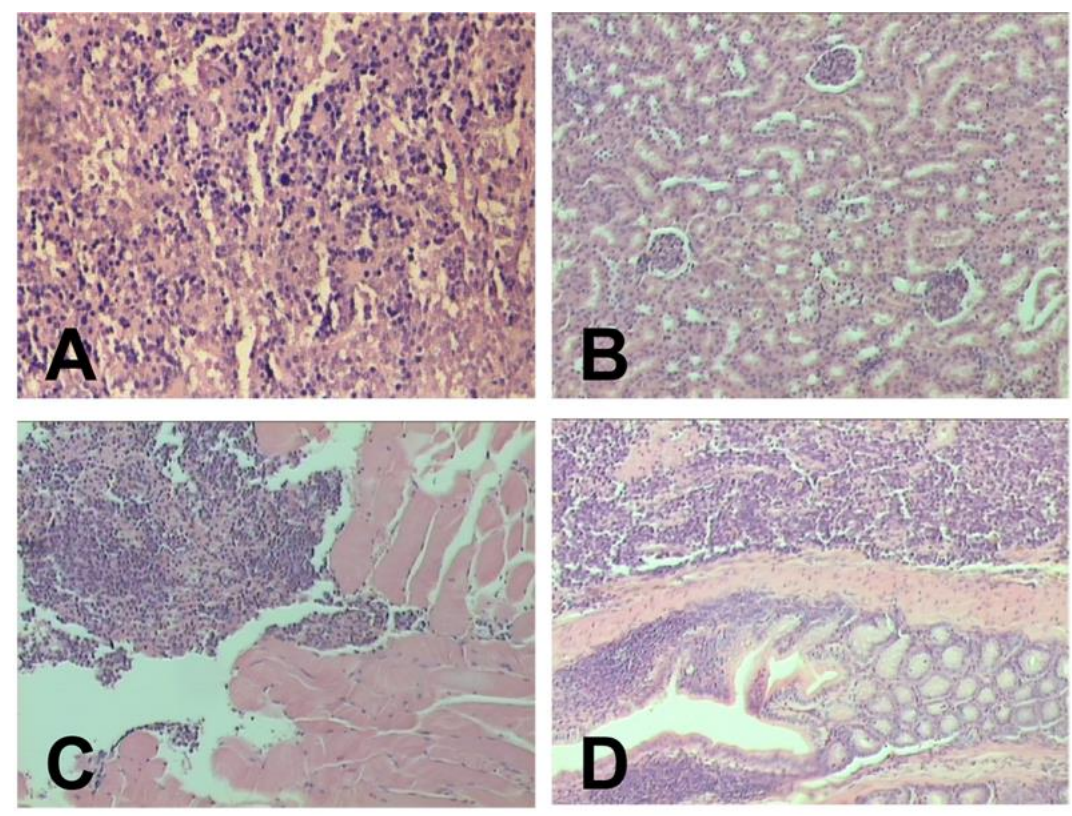

Figure 2. HE staining of primary neuroblastoma (NB) tissues and subcutaneous xenograft tumor tissues. A. HE staining of tumor tissues from a pediatric NB patient (100X). B. HE staining of the subcutaneous xenograft tumor tissues from nude mice inoculated with NB cells from a pediatric NB patient (100X). Metastasis of NB in the muscular tissue (C) and intestinal wall (D) of nude mice inoculated with NB cells from a pediatric patient (100X).

\section{NSE is highly expressed in NB tissues}

NSE is often used as a prognostic indicator of NB (Abdennebi et al., 2000). NSE was highly expressed in the NB tissues collected from the patient and the subcutaneous tumors from the nude mice (Figure 3), showing that the xenograft tumors in the NB mouse model recapitulates what is seen in cells from an NB patient. 

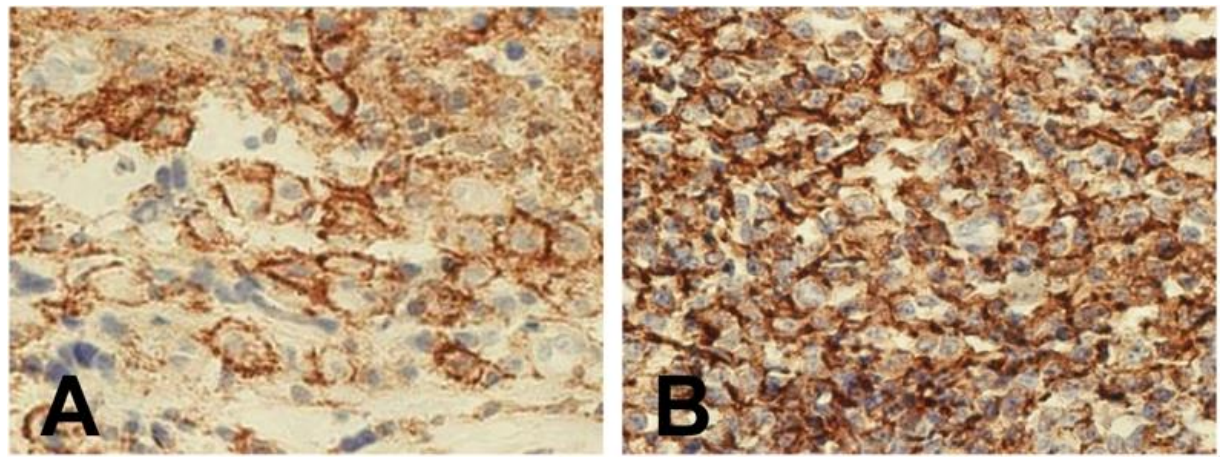

Figure 3. Examination of the expression level of NSE by immunohistochemical analysis. A. Immunohistochemical staining of NSE in the primary tumor tissues from the pediatric NB patient (400X). B. Immunohistochemical staining of NSE in the subcutaneous xenograft tumor tissues from nude mice inoculated with NB cells from a pediatric patient (400X).

\section{NB cells in metastatic tumors exert higher invasive ability}

The primary NB cells exhibit heterogeneity and a subpopulation of cells with high metastatic capability spread to other organs (Figure 1 and Table 1). To examine the invasive ability of tumor cells in NB tissues collected from the patient and cells from metastatic tumors, a Matrigel invasion assay was performed (Figure 4A and C). The NB cell line SH-SY5Y was used as a control (Figure 4B). The invasive ability of metastatic tumor cells was notably higher than that of primary tumor cells and the SH-SY5Y cell line $(P<0.01)$ (Figure 4A). These results suggest that the metastatic NB mouse models established in this study may be useful tools to study the mechanisms of NB metastasis.
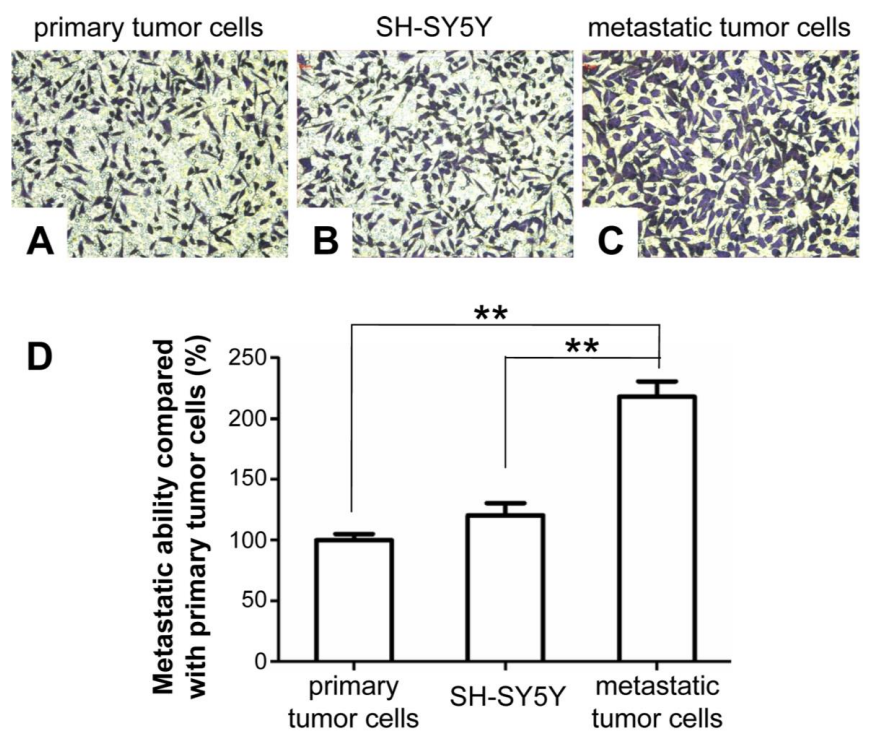

Figure 4. Investigation of the invasive ability of tumor cells in vitro by transwell-based migration assay. The invasive ability of primary tumor cells from the NB patient (A), SH-SY5Y cells (B), and metastatic tumor cells in nude mice (C) were analyzed by an invasion assay $(200 X)$. D. Statistical analysis of the invasion assay $\left({ }^{* \star} P<0.01\right)$. 


\section{Expression levels of metastasis-related genes in metastatic tumors are elevated in the NB mouse model}

To explore the metastatic mechanisms of NB tumors, the expression of metastasisrelated genes was examined by a tumor metastasis microarray. The microarray comparison of gene expression in the primary and metastatic tumors showed that among 113 metastasisrelated candidate genes, 25 genes, including MMP-3, MMP-7, MMP-9, KAI1, RECK, CXCL12, and CXCR4, were found to be significantly upregulated more than 2-fold in metastatic tumors compared to primary tumors (Table 2).

Table 2. Gene expression in the metastatic and primary xenograft NB tumors.

\begin{tabular}{llc}
\hline GenBank ID & Candidate gene & Level in metastatic tumor/Level in primary tumor \\
\hline NM_000610 & CD44 & 1.2432 \\
NM_000609 & CXCL12 & 2.3894 \\
NM_003467 & CXCR4 & 1.9144 \\
NM_002231 & KAl1 & 0.51660 \\
NM_004530 & MMP2 & 1.3320 \\
NM_002422 & MMP3 & 3.9908 \\
NM_004994 & MMP9 & 1.2912 \\
NM_021111 & RECK & 0.59246 \\
NM_003255 & TIMP2 & 1.0827 \\
NM_003376 & VEGF & 4.3672 \\
\hline
\end{tabular}

\section{DISCUSSION}

In this study, the NB cell line isolated from a NB patient was used to inoculate nude mice. The NB model established in this study showed a high xenograft success rate, with a tumor formation rate of $75 \%(36 / 48)$ and a metastasis rate of $21 \%(10 / 48)$ (Table 1). Tumors grew steadily, providing an ideal model for the study of tumor growth, invasion, and metastasis. The primary and metastatic tumors from the established NB nude mouse model were analyzed using light microscopy and immunohistochemistry (Figures 1 and 2). The results showed that the xenograft tumors displayed the same histological structures and immunological properties as the original tumor that was isolated from the patient, demonstrating that tumors that grew in the nude mice faithfully represented the human tumor and not a spontaneously occurring tumor in the mice. This indicates the successful establishment of a human NB xenograft model in nude mice. These subcutaneous and metastatic tumor models represent the characteristics of NB developed in the Chinese population. The study of various NB properties, in particular, those related to metastasis, will have an important impact on our understanding of the mechanisms underlying the metastasis of NB in the Chinese population.

Among the tumor-bearing animal models established in the present study, some models developed local tumors with a large size of up to half the body weight of the mouse, but without metastasis. Some models displayed systemic metastasis without tumor growth at the inoculation site. We also observed models with both local tumor growth and metastasis. The different patterns of tumor growth among our animal models are likely due to the polyclonal nature of the cultured tumor cell line (Speleman et al., 2011). Subclonal cell populations with different invasion and metastasis properties may have been present in the same cell line, leading to different tumor formation patterns (Mackinnon et al., 2009). After many years of study on the mechanism of tumor metastasis, Fidler 
(2001) proposed that a tumor is essentially made from polyclonal heterogeneous cells and that the occurrence of metastasis is primarily caused by those cell clones with high metastatic ability that are present in the tumor cell population. This hypothesis has been verified in animal experiments (Yoneyama et al., 2005). In the present study, 48 nude mice were subcutaneously injected with the same cell line derived from a single NB specimen from a pediatric patient. However, there was variability in local and metastatic tumor development in the nude mice. These results suggest that the tumor cells from the same primary tumor cell line exhibit heterogeneity in their biological characteristics since these tumor cells are of a polyclonal origin. This could explain why these tumor cells displayed different levels of metastatic potential.

The heterogeneity of tumor cells affects the process of tumor growth and evolution and is the basis of tumor metastasis. The metastatic potential of tumor cells correlates directly with the heterogeneity of the tumor cells (Huang et al., 2011). A subpopulation of tumor cells can outgrow other subpopulations and obtain a metastatic phenotype. Compared to primary tumor cells, these cells may have unique traits that give them a higher metastatic potential (Ogawa et al., 2001; Yoneyama et al., 2005). Based on this hypothesis, metastasis could be suppressed by blocking the activity of specific targets. Thus, establishing tumor cell lines from metastatic loci is of great importance for the investigation of metastasis-related genes and the determination of the molecular mechanisms underlying metastasis. To address the important result that some nude mice developed large local tumors without metastasis while others showed systemic metastasis without tumor development at the inoculation site, we conducted a microarray analysis of metastasisassociated gene expression using subcutaneous and metastatic tumor samples. A total of 113 tumor metastasis-associated genes were examined, including genes encoding growth factors and their receptors, adhesion molecules, proteases and protease inhibitors, oncogenes, and tumor suppressing genes. By comparing the results from the metastatic and subcutaneous tumor samples, 25 genes with significant differential expression were selected, including upregulated and downregulated genes. It is worth noting that liver invasion was found in all metastatic tumor models. Since clinical NB metastasis occurs mostly in bone marrow and liver, our results coincide with tissue-specific metastasis of NB (Li et al., 2004). According to the results of our microarray study, RECK, VEGF, CXCL12 and CXCR4 were upregulated in metastatic NB (Azar et al., 2012; Rahmah et al., 2012). It has been shown that the incidence rate of bone marrow metastasis increases with elevated expression of CXCL12 in the bone marrow (Ma et al., 2012). These genes are likely to be closely associated with tissue-specific NB metastasis and may be representative of this process.

In future studies, we aim to isolate more stable subpopulations of tumor cells with higher metastatic potential in order to further study the mechanisms of NB metastasis. To accomplish this goal, immune deficient nude mice will be repeatedly inoculated with tumor cells isolated from metastatic loci. Some subpopulations of tumor cells with high metastatic potential will outgrow other subpopulations and become stabilized after eliminating other maladaptive and unstable subpopulations.

In summary, we successfully established NB mouse models with primary and metastatic tumors. These tumors formed with short latency, a high success rate, and stable histopathological and biological characteristics. Moreover, these tumors mimicked the natural process of growth, local invasion and distant metastasis observed in human NB and could facilitate the thorough observation of potential NB treatments. We have demonstrated that the NB cell line isolated in our lab has the capability of tumor formation and metastasis, indicating that potentially metastatic subpopulations are present in this NB cell line. These subpopulations represent the cellular basis 
of tumor metastasis and demonstrate the existence of tumor cell heterogeneity. These results will benefit future studies on the mechanism of NB etiology and metastasis, as well as the identification of new therapeutic methods and novel anti-tumor drugs.

\title{
Conflicts of interest
}

The authors declare no conflict of interest.

\section{ACKNOWLEDGMENTS}

\author{
Research supported by grants from the Natural Science Foundation of China (NSFC \\ \#81272986 to Q. Dong and NSFC \#81472542 to H.T. Lu).
}

\section{REFERENCES}

Abdennebi M, Boussen H, Harzallah L, Daldoul O, et al. (2000). The use of neuron specific enolase in the prognosis and followup of neuroblastoma in children. Results of a retrospective series of 21 patients. Tunis. Med. 78: 106-108.

Ara T and DeClerck YA (2006). Mechanisms of invasion and metastasis in human neuroblastoma. Cancer Metastasis Rev. 25: 645-657.

Azar WJ, Azar SH, Higgins S, Hu JF, et al. (2012). IGFBP-2 enhances VEGF gene promoter activity and consequent promotion of angiogenesis by neuroblastoma cells. Endocrinology 152: 3332-3342.

Cooper EH (1994). Neuron-specific enolase. Int. J. Biol. Markers 9: 205-210.

Fidler IJ (2001). Angiogenic heterogeneity: regulation of neoplastic angiogenesis by the organ microenvironment. J. Nat/. Cancer Inst. 93: 1040-1041.

Gao Q, Dong Q, Lu HT, Luo B, et al. (2004). Establishment of neuroblastoma cell line in vitro. Shandong Yiyao 44: 3-4.

Hao XW, Dong Q, Lu HT, Lv ZH, et al. (2005). The establishment of neuroblastoma mouse model. Chin. J. Pediatric Surg. 26: 372-375.

Huang R, Cheung NK, Vider J, Cheung IY, et al. (2011). MYCN and MYC regulate tumor proliferation and tumorigenesis directly through BMI1 in human neuroblastomas. FASEB J. 25: 4138-4149.

Li Y, Tian B, Yang J, Zhao L, et al. (2004). Stepwise metastatic human hepatocellular carcinoma cell model system with multiple metastatic potentials established through consecutive in vivo selection and studies on metastatic characteristics. J. Cancer Res. Clin. Oncol. 130: 460-468.

Liu YS, Dong Q, Lu HT, Jiang BX, et al. (2011). The expression of functional chemokine receptor CXCR4 is associated with the metastatic potential of human neuroblastoma. Chin. J. Pediatric Surg. 32: 129-134.

Ma M, Ye JY, Deng R, Dee CM, et al. (2012). Mesenchymal stromal cells may enhance metastasis of neuroblastoma via SDF1/CXCR4 and SDF-1/CXCR7 signaling. Cancer Lett. 312: 1-10.

Mackinnon AC, Yan BC, Joseph LJ and Al-Ahmadie HA (2009). Molecular biology underlying the clinical heterogeneity of prostate cancer: an update. Arch. Pathol. Lab. Med. 133: 1033-1040.

Navarro S, Piqueras M, Villamón E, Yáñez Y, et al. (2012). New prognostic markers in neuroblastoma. Expert Opin. Med. Diagn. 6: 555-567.

Nofiele JT and Cheng HL (2014). Establishment of a lung metastatic breast tumor xenograft model in nude rats. PloS One 9: e97950.

Ogawa K, Nakanishi H, Takeshita F, Futakuchi M, et al. (2001). Establishment of rat hepatocellular carcinoma cell lines with differing metastatic potential in nude mice. Int. J. Cancer 91: 797-802.

Rahmah NN, Sakai K, Sano K and Hongo K (2012). Expression of RECK in endothelial cells of glioma: comparison with CD34 and VEGF expressions. J. Neurooncol. 107: 559-564.

Redlinger RE Jr, Mailliard RB and Barksdale EM Jr. (2004). Neuroblastoma and dendritic cell function. Semin. Pediatr. Surg. 13: $61-71$.

Speleman F, De Preter K and Vandesompele J (2011). Neuroblastoma genetics and phenotype: a tale of heterogeneity. Semin Cancer Biol. 21: 238-244.

Wei H, Niu R and Wu FC (1996). Establishment and copy of metastatic human lung carcinoma xenograft nude mice model. Di-San Junyi Daxue Xuebao 18: 484-488. 
Wu BQ, Song J and Fang WG (1989). Study of human tumor xenograft and cancer biology. Beijing Yike Daxue Xuebao 21: 239-241.

Yan J, Yang Q and Huang Q (2013). Metastasis suppressor genes. Histol. Histopathol. 28: 285-292.

Yoneyama H, Hara T, Kato Y, Yamori T, et al. (2005). Nucleotide sequence variation is frequent in the mitochondrial DNA displacement loop region of individual human tumor cells. Mol. Cancer Res. 3: 14-20. 